Exci tat $\mathrm{i}$ on of hel $\mathrm{i}$ cal l $\mathrm{y}$ - $\mathrm{tr}$ apped-ener get $\mathrm{i}$ c- $\mathrm{i}$ on dri ven resi st i ve i nt er change modes wi th i nt ense deut er i um beam i nj ect i on and enhanced effect on beami ons/bul k pl asmas of LHD

\begin{tabular}{|l|l|}
\hline $\begin{array}{l}\text { j our nal or } \\
\text { publ } \mathrm{i} \text { cat } \mathrm{i} \text { on } \mathrm{ti} \mathrm{tl} \text { e }\end{array}$ & Nucl ear Fusi on \\
\hline vol une & 58 \\
\hline number & 8 \\
\hline page $\mathrm{r}$ ange & 082025 \\
\hline year & $2018-06-29$ \\
\hline URL & ht t p: //hdl . handl e. net /10655/00012909 \\
\hline
\end{tabular}




\title{
Excitation of helically-trapped-energetic-ion driven resistive interchange modes with intense deuterium beam injection and enhanced effect on beam ions/bulk plasmas of LHD
}

\author{
T. Bando ${ }^{1}$, S. Ohdachi ${ }^{1,2}$, M. Isobe ${ }^{1,2}$, Y. Suzuki ${ }^{1,2}$, K. Toi ${ }^{2}$, K. \\ Nagaoka $^{1,3}$, H. Takahashi ${ }^{1,2}$, R. Seki ${ }^{1,2}$, X. D. Du ${ }^{4}$, K. Ogawa ${ }^{1,2}$, \\ T. Ido ${ }^{1,3}$, A. Shimizu ${ }^{2}$, T. Ozaki ${ }^{2}$, and LHD Experiment Group \\ ${ }^{1}$ SOKENDAI (The Graduate University for Advanced Studies), 322-6 Oroshi-cho, \\ Toki 509-5292, Japan \\ ${ }^{2}$ National Institute for Fusion Science, National Institutes of Natural Sciences, 322-6 \\ Oroshi-cho, Toki 509-5292, Japan \\ ${ }^{3}$ Graduate School of Science, Nagoya University, Nagoya, 464-8601, Japan \\ ${ }^{4}$ University of California Irvine, Irvine, CA 92697, USA \\ E-mail: bando.takahiro@nifs.ac.jp \\ January 2018
}

\begin{abstract}
Energetic ion driven resistive InterChange modes (EICs) accompanying repeated bursts of the magnetic fluctuations were found in hydrogen campaign of Large Helical Device. The pressure gradient of helically trapped energetic particles, which are mainly produced by perpendicularly injected beams, drives this mode. Recently, perpendicular neutral beam injection (PERP-NBI) systems are upgraded for deuterium plasma campaign. The beam energies of the two PERP-NBIs are increased from 40/40 $\mathrm{keV}$ to $60 / 80 \mathrm{keV}$, respectively. And the injection powers increase from $6 / 6 \mathrm{MW}$ to 9/9 MW, as well. As results of these upgrades of NBIs, the $\beta$ value of helically trapped energetic ions, $\beta_{\mathrm{h} \perp}$, has increased up to $\sim 0.35 \%$ and induced EICs with larger bursts and smaller repetition frequencies. It is found that the threshold of $\beta_{\mathrm{h} \perp}$ to excite EICs increases with deuterium PERP-NBIs. The amplitude of each burst and effect on energetic beam ions become larger when $\beta_{\mathrm{h} \perp}$ is larger. In addition, a large electrostatic potential about $-25 \mathrm{kV}$ is observed when EICs are excited, which is about two times larger than the potential observed in hydrogen campaign. The transient increases of the electron density and temperature in edge regions are clearly observed when the electrostatic potential is formed.
\end{abstract}

Keywords heliotron, LHD, trapped energetic particles, resistive interchange mode, EPdriven instability, neutron, transport, radial electric field

\section{Introduction}

Studies of interactions between energetic particles (EPs) and MHD modes are quite important to realize the fusion reactor since EP pressure gradient driven modes 
such as fishbone modes cause loss of energetic particles. The fishbone instabilities, accompanying quasi-periodical bursts, were observed in PDX Tokamak [1] for the first time as well as observed in many other Tokamaks. The fishbone instability is considered to be the internal kink modes driven by the trapped energetic ions $[2,3]$. The fishbones affect not only the confinement of EPs but also bulk plasma transport. It is reported that changes of impurity transport [4] and the reduction of the electron temperature $[4,5]$ are induced by fishbones. The fishbone instability is concerned with operation scenarios of ITER [6], and its characteristics are being studied intensively. Recently, other types of fishbones, the so-called off-axis fishbones (or energetic particle driven wall modes (EWMs)) and e-fishbones are also reported. Off-axis fishbone modes were found in JET plasmas [7] where central safety factors were fairly larger than unity. Offaxis fishbones are also studied in DIII-D [8] or JT-60U [9] and it is suggested that the off-axis fishbone is related to external kink modes [8]. E-fishbone is a kind of fishbonelike instability observed in DIII-D during off-axis electron cyclotron current drive [10], observed too in FTU [11] and HL-2A [12]. It is considered that e-fishbones are driven by energetic electrons instead of energetic ions.

Large Helical Device (LHD) is the largest Heliotron-type device with superconducting helical coils having winding numbers of 2/10 [13]. The typical major radius is 3.6 $\mathrm{m}$ and the averaged minor radius is $0.6 \mathrm{~m}$. In LHD, plasmas are intensively heated by three tangential (TAN-) NBIs and two perpendicular (PERP-) NBIs, as shown in figure 1. Figure 1 shows arrangement of three TAN-NBIs (green) and two PERP-NBIs (purple). MHD instabilities appeared with bursts of magnetic fluctuations when plasmas are heated by PERP-NBIs in hydrogen experiment campaign of LHD. This mode is called the Energetic particle driven resistive InterChange mode (EIC), because EICs are destabilized by the resonance between the resistive interchange mode having an $m / n$ $=1 / 1$ mode structure and the precession motion of helically trapped ions produced by the PERP-NB injection $[14,15,16]$. The requirement of the resonance between helically trapped EPs and resistive interchange modes to drive EICs is similar to that of fishbones in Tokamaks.

The velocity of the EPs is extremely fast and it is impossible to interact with slow-frequency pressure driven MHD modes. However, the precession frequency of the helically trapped EPs produced by PERP-NBIs is slow enough to interact with the pressure driven modes. In the LHD, EPs having the large perpendicular (to the magnetic field) component of the velocity are trapped in the weak magnetic field region generated by a pair of helical coils and make precession motion along the weak magnetic field region. These helically trapped EPs are considered to cause EICs.

The effect of EICs is also studied in detail in reference [15]. It is reported that EICs induce loss of energetic beam ions and the formation of a large negative electrostatic potential about $-12 \mathrm{kV}$.

Recently, PERP-NBIs which provide helically trapped particles are upgraded for deuterium plasma campaign of the LHD. The beam energies of the two PERP-NBIs are increased from $40 / 40 \mathrm{keV}$ to $60 / 80 \mathrm{keV}$. And the injection powers are increased 
from 6/6 MW to 9/9 MW. These upgrades of NBIs lead to a significant increase of the helically trapped EPs $\beta, \beta_{\mathrm{h} \perp}$.

In this article, characteristics of EICs with deuterium PERP-NBIs are reported. In particular, the stability of the EIC and the effect on energetic beam ions and bulk plasmas with deuterium PERP-NBIs are presented. The experimental condition is the following. The vacuum magnetic axis is $3.6 \mathrm{~m}$, the magnitude of magnetic field at axis is $2.75 \mathrm{~T}$ or $2.85 \mathrm{~T}$, and the aspect ratio is 5.8. The main plasma parameters in this study are summarized in table 1.

In section 2, characteristics of excitations of EICs are studied in comparison with the case of hydrogen PERP-NBIs. The dependence of initial frequencies on the injection energies of PERP-NBIs is discussed there. It is then shown that $\beta_{\mathrm{h} \perp}$ is a good indicator for the excitation of EICs and the threshold value is clearly higher in the deuterium case. The enhanced effects on EPs and bulk plasmas by EICs are discussed in section 3. In section 4, the stabilities of EICs with deuterium PERP-NBIs are discussed. Then the summary is given in section 5 .

It is noted that the same data set is used for figures 6, 7, 9, and 11 where PERPNBIs use deuterium gas and TAN-NBIs use hydrogen gas. And, ECR injection is not used because ECR heating is considered to stabilize EICs [16]. RMPs are not applied to the plasma because RMPs are considered to affect the stability of interchange modes in LHD [18].

\section{Excitation of EICs with enhanced deuterium beams}

\subsection{Increase of the energetic particle pressure with deuterium beams}

In order to discuss the excitation condition of EICs, the pressure of EPs should be evaluated. The pressure of helically trapped EPs normalized by the magnetic field pressure, $\beta_{\mathrm{h} \perp}$, can be estimated by

$$
\beta_{\mathrm{h} \perp}=\beta_{\text {dia. }}-\beta_{\text {bulk }} .
$$

Here, $\beta_{\text {dia. }}$ is the total $\beta$ value estimated with the diamagnetic loop [19] and $\beta_{\text {bulk }}$ is the contributions from the bulk plasmas obtained by Thomson scattering measurement [20]. $\beta_{\text {dia. }}$ is estimated by $\frac{4}{3} \mu_{0} W p_{\text {dia. }} /\left(V p B_{\text {ave }}^{2}\right) . W p_{\text {dia. }}$ is the plasma stored energy estimated with the diamagnetic loop using the JDIA code [21]. $V p$ is the plasma volume in the vacuum configuration. $B_{\text {ave }}$ is the averaged toroidal field in the vacuum configuration. $T_{\mathrm{e}}=T_{\mathrm{i}}$ and $n_{\mathrm{e}}=n_{\mathrm{i}}$ are assumed to calculate $\beta_{\text {bulk }}$.

Note that the diamagnetic loop measures $\beta_{\text {dia. }}$ determined by the perpendicular pressure $p_{\perp}[22]$. Though the mechanism about contributions to $p_{\perp}$ by helically trapped EPs from PERP-NBIs and passing EPs from TAN-NBIs should be investigated to use $\beta_{\mathrm{h} \perp}$ as the pressure of helically trapped EPs, it is not easy to discuss the detail. However, $p_{\perp}$ seems to be mainly contributed by helically trapped EPs from following two observations. First, a previous simulation study [23] using a Mote Carlo code in LHD revealed that the contribution to $p_{\perp}$ by tangential beams is $10 \%$ of that to the tangential 
pressure $p_{\|}$. This suggests that passing EPs provided by TAN-NBIs contribute to $p_{\|}$. Second, when PERP-NBIs are injected, $\beta_{\text {dia. }}$ increases significantly while $\beta_{\text {bulk }}$ does not increase like $\beta_{\text {dia. }}$ as seen with figure 2 (a) in the next paragraph. Because PERPNBIs provides helically trapped EPs, the increased $\beta_{\text {dia. }}$ should be due to the increased pressure of helically trapped EPs. From these observations, $p_{\perp}$ should be contributed by mainly helically trapped EPs. Therefore, the pressure of helically trapped EPs may be estimated by equation 1 .

Figure 2 (a) shows the time evolution of $\beta$ value for hydrogen PERP-NBI (blue) and deuterium PERP-NBI (red) cases. The black solid line and the black dashed line indicate the pulse pattern of $\operatorname{PERP}-\mathrm{NBI}(\# 4)$ and $\operatorname{PERP}-\mathrm{NBI}(\# 5)$, respectively. The timing when PERP-NBs are injected is the same in both cases. EICs are typically observed in the so-called "High-Ti" type discharges, where carbon pellets are used to achieve higher ion temperature [24]. The sudden increase of the $\beta_{\text {bulk }}$ around $4.57 \mathrm{~s}$ is caused by a carbon pellet injection. The ablation of the carbon pellet can cause the increase of the total pressure with the increase of the electron density and the decrease of the electron temperature. The injection of PERP-NBI(\#4) starts at $4.6 \mathrm{~s}$, and then the $\beta_{\text {dia. }}$ starts to increase. Though $\beta_{\text {bulk }}$ does not change, $\beta_{\text {dia. }}$ increases clearly in the deuterium case. Therefore, the increase of $\beta_{\text {dia. }}$ is mainly caused by the increase of $\beta_{\mathrm{h} \perp}$. The increase of $\beta_{\mathrm{h} \perp}$ is about $\sim 0.35 \%$ so the pressure of helically trapped EPs to drive EICs seems to increase in the deuterium case.

An example of profiles of $T_{\mathrm{i}}, T_{\mathrm{e}}$, and $n_{\mathrm{e}}$ is shown in figure 3 . As seen in figure $3(\mathrm{a})$, the assumption that $T_{\mathrm{e}}=T_{\mathrm{i}}$ can be violated with High-Ti discharges. However, if the total energy is calculated by integrating the energy density on a plasma of LHD using $T_{\mathrm{i}}, T_{\mathrm{e}}$, and $n_{\mathrm{e}}$ profiles in figure 3 as a reference case assuming $n_{\mathrm{e}}=n_{\mathrm{i}}$, the contribution of ions and electrons to the total energy is about $60 \%$ and about $40 \%$, respectively. Typical error in the estimation of the $\beta_{\mathrm{h} \perp}$ is about $15 \%$ with these contributions. Therefore, $T_{\mathrm{e}}=T_{\mathrm{i}}$ seems not to be unreasonable. $n_{\mathrm{e}}=n_{\mathrm{i}}$ should be also violated after the carbon pellet injection. However, the measurement of $Z_{\text {eff }}$ is not easy in LHD and the assumption, $n_{\mathrm{e}}=n_{\mathrm{i}}$, is used here.

Figure 4 shows the magnetic fluctuations when EICs are excited with hydrogen PERP-NBIs (blue) and deuterium PERP-NBIs (red). In the deuterium case, $\beta_{\mathrm{h} \perp}$ is higher compared to the hydrogen case and there are magnetic fluctuations $2 \sim 5$ times higher observed if EICs are excited showing a longer interval between events as shown in figure 4. The mode number of the EICs in the deuterium and hydrogen experiment is $m / n=1 / 1$ [15]. These observations suggest that the characteristics of EICs are basically not changed. However, the amplitude of the mode is drastically enhanced with upgraded PERP-NBIs whose injection power is higher.

In deuterium experiments, larger amplitudes with smaller repetition frequencies lead to larger EP losses as indicated by green dashed circles in figure 2 (a). The large EP loss reduces the EP pressure gradient at the mode rational surface, i.e., the $\iota=$ 1 rational surface. Therefore, it is thought that longer time is required to reach the threshold of $\beta_{\mathrm{h} \perp}$ to excite EICs. On the other hand, since the loss of EPs by each EIC is 
smaller in the hydrogen case as shown in figure 2, the repetition frequency of EICs may become smaller, though amplitudes of magnetic fluctuations by EICs are comparable or smaller in the hydrogen case.

\subsection{Dependence of initial frequencies on injection energies}

Figure 5 (a) shows an example of magnetic fluctuations when an EIC is excited. The frequency chirping down from $12 \mathrm{kHz}$ to $3.6 \mathrm{kHz}$ is observed in this case. Because the injection energies are different between hydrogen PERP-NBIs and deuterium PERPNBIs, the initial frequencies with different injection energies can be compared. Figure 5 (b) shows the observed initial frequency with different injection energies of the hydrogen PERP-NBIs and the deuterium PERP-NBIs. The initial frequencies with injection energies around $40 \mathrm{keV}$ are $7 \sim 8.3 \mathrm{kHz}$, and the initial frequencies with injection energies around $60 \mathrm{keV}$ and $66 \mathrm{keV}$ are $8.5 \sim 12 \mathrm{kHz}$. The poloidal precession frequencies of the helically trapped EPs at several major radii are estimated by calculating EP orbits at $45 \mathrm{keV}$ ions and at $66 \mathrm{keV}$ deuterons as shown in figure 5 (c). In this calculation, the magnitude of the magnetic field at the axis is $3 \mathrm{~T}$ and the initial pitch angle of EPs is -0.031 . The pitch angle is defined as $\cos \theta$. Here, $\theta$ is the angle between the magnetic field line and the direction of the EP motion. The pitch angle -0.031 is corresponding to $\theta=0.51 \pi$ radian and almost perpendicular to the magnetic field. EPs provided by PERP-NBIs have such large perpendicular component as mentioned in section 1 . The $\iota$ profile of the vacuum configuration calculated by VMEC code [25] is shown in figure $5(\mathrm{~d})$. The $\iota=1$ rational surface is located around $R=4.3 \mathrm{~m}$ at the horizontally elongated section. The corresponding poloidal precession frequencies are $7.5 \mathrm{kHz}$ and $12.4 \mathrm{kHz}$ for the ion and the deuteron, respectively. The error bar in the horizontal axis indicates the difference of orbit deviation difference from magnetic surfaces in the major radius and the error bar in the vertical axis indicates the difference in the pitch angles difference which are assumed in the orbit trace calculations. The range of the error bar in the vertical axis comes from pitches between 0.31 and -0.31 . It is found that the poloidal precession frequencies are almost the same as the initial frequencies in figure 5 (b). Taking into account that EICs are excited with PERP-NBIs, the agreement on the frequencies clearly support that EICs are excited by helically trapped EPs around the $\iota=1$ rational surface in the peripheral region.

\subsection{Increased threshold of EP pressure to excite EICs}

In the hydrogen campaign, it was found that EICs are excited only in relatively lower electron density regions, $\bar{n}_{\mathrm{e}}<1.5 \times 10^{19} \mathrm{~m}^{-3}[15]$. Therefore, it is reasonable to discuss about the stability as a function of the electron density. Figure 6 shows the relationship of the line averaged electron density, measured by the FIR interferometer [26], and $\beta_{\mathrm{h} \perp}$ when EICs are excited. Results with hydrogen PERP-NBIs (blue triangles) and deuterium PERP-NBIs (red dots) are compared. $\beta_{\mathrm{h} \perp}$ is found to increase as the electron density increases. In addition, there is a clear difference between the $\beta_{\mathrm{h} \perp}$ 
value in deuterium and hydrogen cases at the same electron density. For example, $\beta_{\mathrm{h} \perp}$ is about $0.7 \%$ in the deuterium case and about $0.4 \%$ in the hydrogen case around $\bar{n}_{\mathrm{e}} \sim 1.7 \times 10^{19} \mathrm{~m}^{-3}$ in figure 6 . Besides, EICs are not excited when $\beta_{\mathrm{h} \perp}$ is smaller than the threshold $\beta_{\mathrm{h} \perp}$ about $\bar{n}_{\mathrm{e}} \sim 1.7 \times 10^{19} \mathrm{~m}^{-3}$. These observations indicate that the threshold of the EP pressure to drive EICs becomes higher with deuterium PERPNBIs. In figure 6 , the length of vertical bars with some red dots indicate the decrease of $\beta_{\mathrm{h} \perp}$ assuming the experimentally obtained $T_{\mathrm{i}}$ and $T_{\mathrm{e}}$ profiles such as profiles in figure 3. Even if the vertical bars are considered, the difference of $\beta_{\mathrm{h} \perp}$ between hydrogen and deuterium cases can be seen. Note that EICs are not observed with larger electron density, $\bar{n}_{\mathrm{e}}>1.9 \times 10^{19} \mathrm{~m}^{-3}$, as indicated by grey circles for deuterium and grey triangles for hydrogen in figure 6 . This observation indicates that there is the threshold of the electron density as well as the threshold of $\beta_{\mathrm{h} \perp}$.

Excitation of EICs may be dependent on the pressure gradient of bulk plasma and EP pressure. One possible explanation of the different thresholds is the pressure gradient of the bulk plasma. The electron pressure gradient, which drives resistive interchange modes, is thereby investigated. Evaluation of the magnetic Reynold's number around the $\iota=1$ surface is also performed because it has been shown that the saturation level of the mode amplitude of resistive interchange modes is proportional to $S^{-1 / 3}$ in LHD [27]. Here, $S$ is the magnetic Reynold's number.

Figure 7 shows (a) the electron pressure gradient and (b) the magnetic Reynold's number with the same data set shown in figure 6 . It is observed that the electron pressure gradient and the magnetic Reynold's number are almost the same between hydrogen and deuterium cases. Therefore, it is certain that the stability of the bulk resistive interchange mode is not changed and the stability of EICs is mainly determined by the EP pressure.

\subsection{Larger radial mode structure of EICs}

In the hydrogen campaign, the radial mode structure of EICs measured by ECE measurement [28] is localized around $\iota=1$ rational surfaces. The eigenfunction of the mode is a type of the tearing parity type (opposite phase across the rational surface) $[14,15]$. The normalized mode width $w / a$ is about 0.065 and the radial displacement $\xi_{r}$ is about $0.5 \mathrm{~cm}$ [15]. Here, $w$ is the mode width and $a$ is the averaged plasma minor radius. $\xi_{r}$ is defined as $\tilde{T}_{e} / \nabla T_{e, e q}$ assuming that plasmas are incompressible, i.e. $\nabla \cdot \xi=0 . \tilde{T}_{e}$ is the fluctuation component of the electron temperature induced by EICs and $\nabla T_{e, e q}$ is the electron temperature gradient.

The radial mode structure of EICs is measured in the case of the deuterium campaign. Figure 8 shows time evolution of (a) the magnetic fluctuations and (b) the radial profile of the $T_{e}$ fluctuation component measured by ECE when an EIC is excited. Here, Singular Spectrum Analysis (SSA) [29] is used to obtain the fluctuation component of ECE signals. SSA is a technique based on Singular Value Decomposition (SVD) and is effective for separating the fluctuation component from signals if the trend 
component is also changing. When the EIC is excited, the mode structure is extended from the plasma edge to the middle of the plasma around $4.7445 \mathrm{~s}$ in figure 8 . The reason of the extension to the middle of the plasma has not been clarified. After the extended structure to the middle of the plasma disappears, the oscillations having tearing parity localized around the $\iota=1$ surface are observed in deuterium experiment, as well. In the deuterium case, $w / a$ is about 0.15 evaluated from figure $8(\mathrm{~b})$. And $\xi_{r}$ is about $1.5 \mathrm{~cm}$. These $w / a$ and $\xi_{r}$ are larger than those in the hydrogen case [15]. It is consistent with the observation that the maximum amplitude of magnetic fluctuations with the EIC is larger than the amplitude in hydrogen experiment.

\section{Enhanced effect of EICs on EPs and bulk plasmas}

In this section, the effect of EICs on EPs and bulk plasmas are discussed. In section 2.3, it is shown that the threshold of $\beta_{\mathrm{h} \perp}$ to excite EICs increases with deuterium PERPNBIs. Furthermore, it is found that the maximum amplitudes of magnetic fluctuations normalized by the toroidal magnetic field $B_{\mathrm{t}}, \delta b_{\theta} / B_{\mathrm{t}}$, observed with EICs increase as $\beta_{\mathrm{h} \perp}$ increases as shown in figure 9 . Here, the definition of $\delta b_{\theta} / B_{\mathrm{t}}$ is shown schematically in figure 10 (b). It is observed that the effect of EICs on EPs increase with larger $\delta b_{\theta} / B_{\mathrm{t}}$ as discussed below.

\subsection{Effect of EICs on EPs}

The total neutron emission rate, $S_{\mathrm{n}}$, is a good indicator of the density of helically trapped EPs if PERP-NBIs only use deuterium gas and TAN-NBIs only use hydrogen gas, because the total emission rate is dominated by the beam-plasma interactions. The total neutron emission rate is obtained by the newly installed Neutron Flux Monitor (NFM) $[30,31]$. It should be noted that the time response of NFM is about $2 \mathrm{~ms}$. Therefore, detailed process of the reduction of the neutron emission rate cannot be studied by this signal. Figure 10 shows the time evolution of (a) the total neutron emission rate and (b) magnetic fluctuations with EICs if PERP-NBIs only use deuterium gas. As shown in figure 10, the neutron emission rate drops rapidly by EICs and recovers slowly. The drop rate of the neutron emission rate, $-d S_{\mathrm{n}} / S_{\mathrm{n}}$, is higher as $\delta b_{\theta} / B_{\mathrm{t}}$ increases, see figure 11 (a). Observed drop rate is up to about $55 \%$. This tendency is consistent with larger losses or EP re-distributions during events with larger $\delta b_{\theta} / B_{\mathrm{t}}$. Figure 11 (b) shows the maximum of $-d W p_{\text {dia. }} / d t$ just after EICs. Here, $d W p_{\text {dia. }} / d t$ is the time derivative of the plasma stored energy measured by the diamagnetic loop [19]. $d W p_{\text {dia. }} / d t$ seems to be caused by EPs losses because the change of the stored energy of the bulk plasma before and after an EIC is small (measured by Thomson scattering measurement [20]). Plasma stored energy decreases as $\delta b_{\theta} / B_{\mathrm{t}}$ increases suggesting an enhancement of EPs losses. 


\subsection{Effect of EICs on bulk plasmas}

The electrostatic potential profile can be obtained from HIBP measurement [32] in LHD. The HIBP measurements discussed in this section are conducted in a relatively lower density region, $\bar{n}_{\mathrm{e}}<1 \times 10^{19} \mathrm{~m}^{-3}$, where HIBP measurements have good signal to noise ratio. The obtained time evolution of the electrostatic potential is shown in figure 12 (b). Here, the signal from HIBP measurement is averaged in order to reduce high frequency noise. The time window for the averaging is about $0.1 \mathrm{~ms}$.

Figure 12 (c) shows the electrostatic potentials in different radial positions obtained with HIBP measurement. Formations of large negative potential about $-25 \mathrm{kV}$ is observed in the core region, which is about two times larger than that observed in the hydrogen case [15]. The solid and dashed red lines are calculated by integrating $E_{r}=-a \exp \left\{-\left[\frac{\rho-0.85}{0.15}\right]^{2}\right\} \mathrm{kV} \mathrm{m}^{-1}$ along the radius, which is as same as the function used in the reference [15], so that the two profiles can be compared. $a$ is a factor which determines the depth of the potential. The dashed red line indicates the extrapolation assuming the potential increases monotonically. The change of the potential around $\rho \sim 0.75$ suggests that EPs are expelled by EICs from around the $\iota=1$ rational surface and the large electric field shear is formed in the edge region.

It is noted that the amplitude of $\delta b_{\theta} / B_{\mathrm{t}} \sim 1.5 \times 10^{-4}$ observed in these experiments with HIBP measurement is relatively smaller than in the typical deuterium experiment because the HIBP measurement is possible only in the low electron density experiments. The electric potential formed may be deeper if the electron density and the $\delta b_{\theta} / B_{\mathrm{t}}$ are larger.

When the electrostatic potential is formed, there is a transient increase of the electron density and temperature in the edge region. Figure 13 shows the time evolution of (a) magnetic fluctuations, (b) the electrostatic potential, (c) the electron density in the edge region, and (d) the electron temperature in the edge region. The increase of the electron density and the electron temperature are clearly observed while the electrostatic potential is formed. It is possible that the particle transport is affected by the generation of the electric field shear [33].

As seen in figure 12 (a), oscillations caused by EICs seem to disappear when the potential recovers. From this observation, a possible mechanism how the frequency of the EIC is determined is discussed. It is found that the frequency of EICs is higher when the electrostatic potential is deeper. In LHD, the observed frequency of resistive interchange modes is found to be sum of the electron diamagnetic drift frequency and the $E \times B$ drift frequency [34]. It is reasonable that the rotation frequency is related with the amplitude of the potential, which may increase as the strength of $E_{r}$ formed by EICs increases. 


\section{Discussion}

As discussed in section 2.3, the threshold of the EP pressure required to excite EICs is larger with deuterium PERP-NBIs. The threshold of the EP pressure also depends on the electron density and EICs are stable if $\bar{n}_{\mathrm{e}}>1.9 \times 10^{19} \mathrm{~m}^{-3}$. Here, reasons for the increased threshold of the EP pressure and the threshold of the electron density are discussed separately.

The increased threshold of the EP pressure is not due to the change of the stability of resistive interchange modes for hydrogen and deuterium cases because the electron pressure gradient and the magnetic Reynold's number are similar as shown in figure 7. One of the candidates to explain this stabilization of EICs is that orbits of the perpendicularly injected deuterium beams deviate more largely from the magnetic surfaces. This larger deviation is caused by the larger energy of deuterium beams. If the orbit deviation is larger, the interaction with the MHD mode localized at the rational surface thereby becomes smaller. In other words, the large deuterium EP pressure compared to the hydrogen case is necessary to excite EICs. This is one possible explanation why EICs with deuterium beams require larger EP pressure.

The reasons for dependence of the threshold of $\beta_{\mathrm{h} \perp}$ on the electron density and stabilization of EICs where $\bar{n}_{\mathrm{e}}>1.9 \times 10^{19} \mathrm{~m}^{-3}$ have not been clarified. One possible reason is the change of $d\left(\beta_{\mathrm{h} \perp}\right)_{\text {loc. }} / d \rho$ around the $\iota=1$ rational surface, which is the

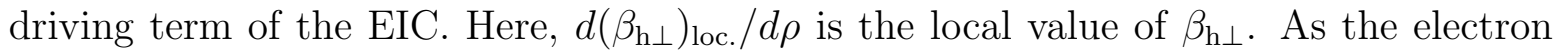
density increases, the relaxation time of helically trapped EPs around the $\iota=1$ rational surface decreases. Therefore, $d\left(\beta_{\mathrm{h} \perp}\right)_{\text {loc. }} / d \rho$ around the $\iota=1$ rational surface perhaps decreases with large electron densities.Other possible reason is modifications of the EP pressure by Alfven eigen modes (AEs). Toroidal Alfven eigen modes (TAEs) are commonly observed in magnetic confined devices. In this study, TAEs seem not to be excited because the frequencies of TAEs [35, 36] in LHD are usually much higher than that of EICs $(<=15 \mathrm{kHz})$. Besides, the magnitude of the magnetic field at the magnetic axis is less than $2 \mathrm{~T}$ where TAEs are observed in LHD while the magnitude is $2.75 \mathrm{~T}$ or $2.85 \mathrm{~T}$ in this study. However, the large EP beta or the large $\beta_{\text {bulk }}$ can be realized with the large electron density and can cause beta-induced Alfven eigen modes (BAEs) or beta-induced Alfven-acoustic eigen modes (BAAEs), which frequencies can be lower than those of TAEs. The possibility of excitations of BAEs or BAAEs should be examined in the future.

Here, we have briefly evaluated the danger of EICs in future reactor devices. The stability of EICs is roughly estimated using parameters expected in the Heliotron-based fusion reactor FFHR-d1 [37], which is a design for helical fusion DEMO reactors. FFHRd1 has the robust similarity with LHD. The case of "IS-SA-PD" in table 1 of reference [37] is used, which has a quite similar magnetic configuration studied in this article. The $\beta$ value of $\alpha$ particles is around $0.1 \%$ where the magnetic Reynold's number is about $10^{9}$. These values are large enough to excite EICs according to observations in section 2.3. Therefore, it is possible that EICs can be excited and will affect the confinement 
of EPs in the future Heliotron-based reactor devices. Further studies regarding effect of EICs on confinement of alpha particles and alpha heating will be examined in the future. Comparison about loss of EPs in FFHR-d1 between loss by EICs and loss by relaxation process is also required.

\section{Summary}

In this article, the excitation of EICs with intense deuterium PERP-NBIs and the effect of EPs/bulk plasma parameters on EICs stability are reported. The beam energies and injection powers of PERP-NBIs in LHD are increased for the deuterium plasma campaign. Different initial frequencies of EICs are observed for different injection energies of PERP-NBIs. These initial frequencies are similar to the calculated poloidal precession frequencies of helically trapped particles. This observation confirms the scenario in which EICs are driven by helically trapped EP pressure. The threshold of EP pressure to excite EICs appear to increase with deuterium PERP-NBIs although the stability of resistive interchange modes is not changed. A candidate to explain the stabilization of EICs is the larger orbit deviation of helically trapped ions as discussed in section 4. It is observed that the effect on energetic beam ions is more significant if $\beta_{\mathrm{h} \perp}$ is larger. In addition, a large electrostatic potential about $-25 \mathrm{kV}$ is formed when EICs are excited, which is about two times larger than the potential observed in the hydrogen campaign. The transient increase of the electron density and temperature in edge regions is clearly observed when the electrostatic potential is formed.

\section{Acknowledgments}

The authors would like to thank Prof. Y. Todo, Dr. H. Wang, and Dr. S. Nishimura for discussions regarding theoretical interpretations of EICs. Helpful comments for analysis by Prof. K. Y. Watanabe, Prof. S. Sakakibara, Prof. J. Miyazawa, Dr. S. Yamamoto, and Dr. K. Saito are greatly appreciated.

This work was supported by NIFS budget code ULPP028 and is also partially supported by the Ministry of Education, Science, Sports and Culture Grant-in-Aid for Scientific Research 26249144, by the JSPS-NRF-NSFC A3 Foresight Program (NSFC: No.11261140328, NRF: No. 2012K2A2A6000443).

[1] McGuire K. 1983 Phys. Rev. Lett. 50891

[2] Chen L. and White B. R. 1984 Phys. Rev. Lett. 521122

[3] Coppi B. and Porcelli F. 1986 Phys. Rev. Lett. 572272

[4] Cecconello M. et al 2015 Nucl. Fusion 55032002

[5] Günter S. et al 1999 Nucl. Fusion 391535

[6] Fasoli A. et al 2007 Nucl. Fusion 47 S264

[7] Huysmans A. T. G. 1999 Nucl. Fusion 391489

[8] Okabayashi M. et al 2011 Phys. Plasma 18056112

[9] Matsunaga G. et al 2010 Nucl. Fusion 50084003 
Excitation of helically-trapped-energetic-ion driven resistive interchange modes with intense deuterium beam

[10] Wong L. K et al 2000 Phys. Rev. Lett. 85996

[11] Guimarães-Filho O. Z. et al 2012 Nucl. Fusion 52094009

[12] He Hongda 2014 Nucl. Fusion 54114001

[13] Komori A. et al 2010 Fusion Sci. Technol. 581

[14] Du X. D. et al 2015 Phys. Rev. Lett. 114155003

[15] Du X. D. et al 2016 Nucl. Fusion 56016002

[16] Du X. D. et al 2017 Phys. Rev. Lett. 118125001

[17] Takahashi H. et al 2017 Presented at 21st International Stellarator-Heliotron

[18] Sakakibara S. et al 2006 33rd EPS Conference on Plasma Phys. Rome, 19-23 June 2006 ECA 301 P-4.113

[19] Sakakibara S. et al 2010 Fusion Sci. and Technol. 58471

[20] Yamada I. et al 2016 Rev. Sci. Instrum. 87 11E531

[21] Yamaguchi T. et al 2006 Plasma Phys. Control. Fusion 48 L73

[22] Pustovitov V. D. 2010 Plasma Phys. Control. Fusion 52065001

[23] Seki R. et al 2010 Plasma and Fusion Research 5027

[24] Nagaoka K. et al 2015 Nucl. Fusion 55113020

[25] Hirshman P. S. and Whitson C. J. 1983 Phys. Fluids 263553

[26] Akiyama T. et al 2010 Fusion Sci. Technol. 58352

[27] Sakakibara S. 2008 Plasma Phys. Control. Fusion 50124014

[28] Tokuzawa T. et al 2010 Fusion Sci. and Technol. 58364

[29] Golyandina Nina and Zhigljavsky Anatoly 2013 Singular Spectrum Analysis for time series (Springer Science Business Media)

[30] Isobe M. et al 2014 Rev. Sci. Instrum. 85 11E114

[31] Nishitani T. et al 2017 Fusion Eng. Des. 1231020

[32] Ido T. et al 2006 Rev. Sci. Instrum. 77 10F523

[33] Moyer A. R. 1995 Phys. Plasma 22397

[34] Takemura Y. et al 2013 Plasma and Fusion Research 81402123

[35] Yamamoto S. et al 2005 Nucl. Fusion 45326

[36] Ogawa K. et al 2013 Nucl. Fusion 53053012

[37] Miyazawa J. et al 2014 Nucl. Fusion 54043010

\begin{tabular}{c|c}
\hline Major radius & $3.6 \mathrm{~m}$ \\
\hline Averaged minor radius & $0.6 \mathrm{~m}$ \\
\hline Magnitude of magnetic field at axis & $2.75 \mathrm{~T} \mathrm{or} 2.85 \mathrm{~T}$ \\
\hline$T_{\mathrm{i} 0}$ & $\sim 10 \mathrm{keV}[17]$ \\
\hline$T_{\mathrm{e} 0}$ & $\sim 3.5 \mathrm{keV}$ \\
\hline$n_{\mathrm{e} 0}$ & $0.5 \times 10^{19} \mathrm{~m}^{-3} \sim 2.0 \times 10^{19} \mathrm{~m}^{-3}$ \\
\hline
\end{tabular}

Table 1. Main plasma parameters in this study. 
Excitation of helically-trapped-energetic-ion driven resistive interchange modes with intense deuterium beam

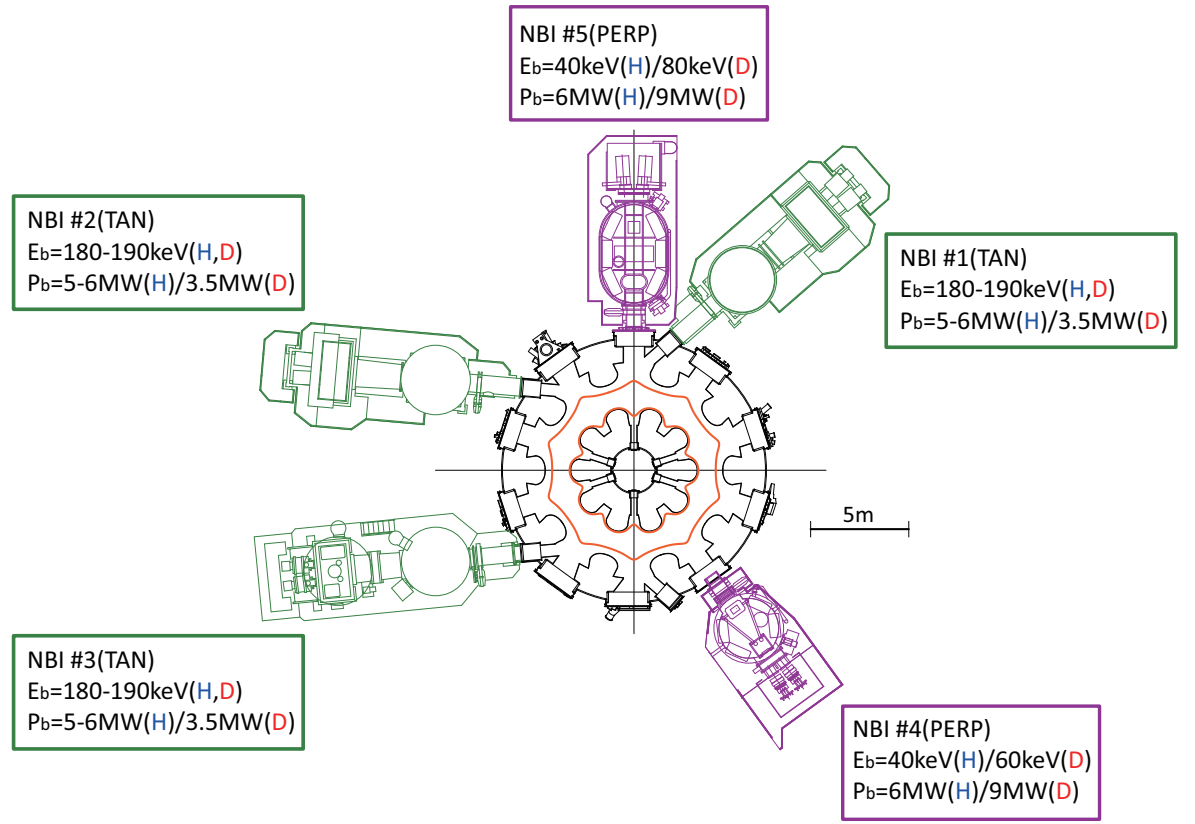

Figure 1. Configuration of LHD and NBIs. Three TAN-NBIs (green) and two PERPNBIs (purple) are shown. The injection energies and powers of 5 NBIs are shown regarding hydrogen $(\mathrm{H})$ and deuterium $(\mathrm{D})$ cases. 


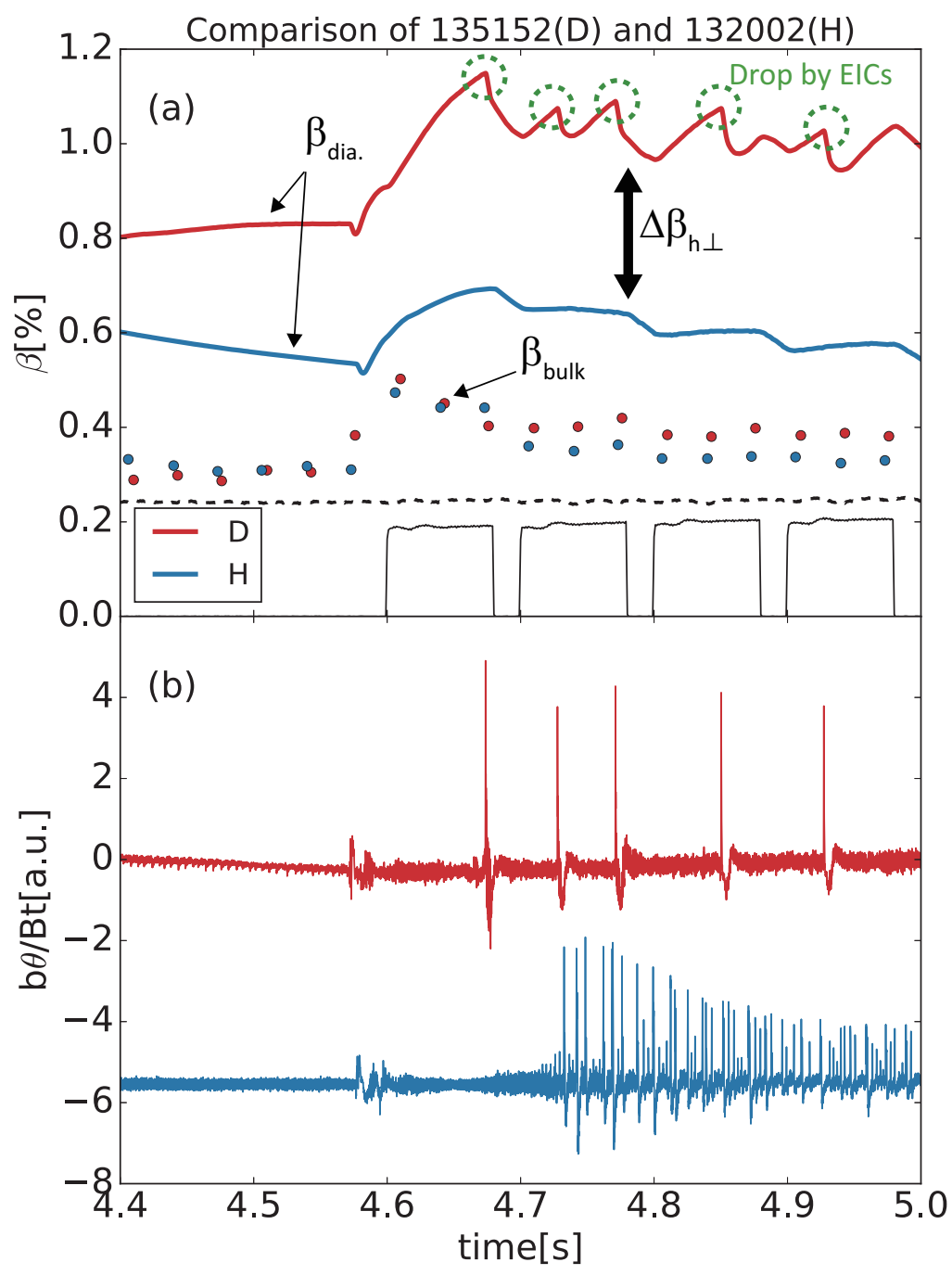

Figure 2. Figure (a) shows comparison of $\beta$ values between plasmas heated by hydrogen PERP-NBIs (blue) and deuterium PERP-NBIs (red) is shown. The solid lines indicate $\beta_{\text {dia. }}$ and the points indicate $\beta_{\text {bulk. }}$. The black solid line and the black dashed line indicate the pulse pattern of PERP-NBI(\#4) and PERP-NBI(\#5), respectively. The green dashed circles indicate drops of $\beta_{\text {dia. }}$ by EICs. Figure (b) shows comparison of magnetic fluctuations. Bursty magnetic fluctuations of EICs are observed in both cases. Here, the electron densities in the hydrogen case and the deuterium case are almost the same. 




Figure 3. Profiles of (a) the electron temperature and the ion temperature, and (b) the electron density in a high-Ti discharge. 


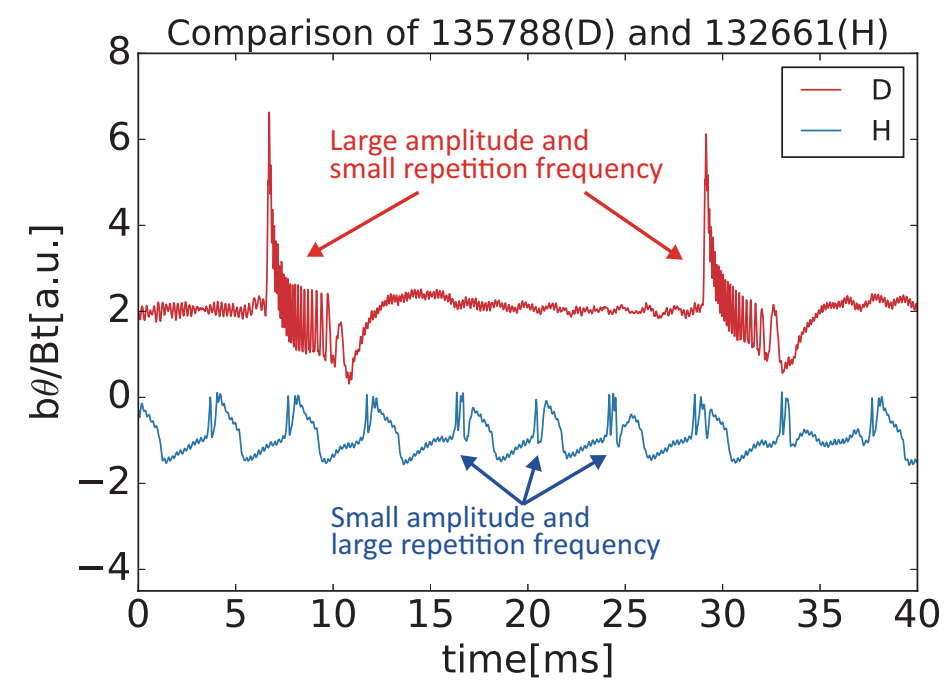

Figure 4. Magnetic fluctuations when EICs are excited with hydrogen PERP-NBIs (blue) and deuterium (red) PERP-NBIs are shown. The larger and less frequent magnetic fluctuations are observed in the deuterium case. Here, the electron densities in the hydrogen case and the deuterium case are almost the same. The scales of y-axis in this figure and figure 2 (b) are the same.
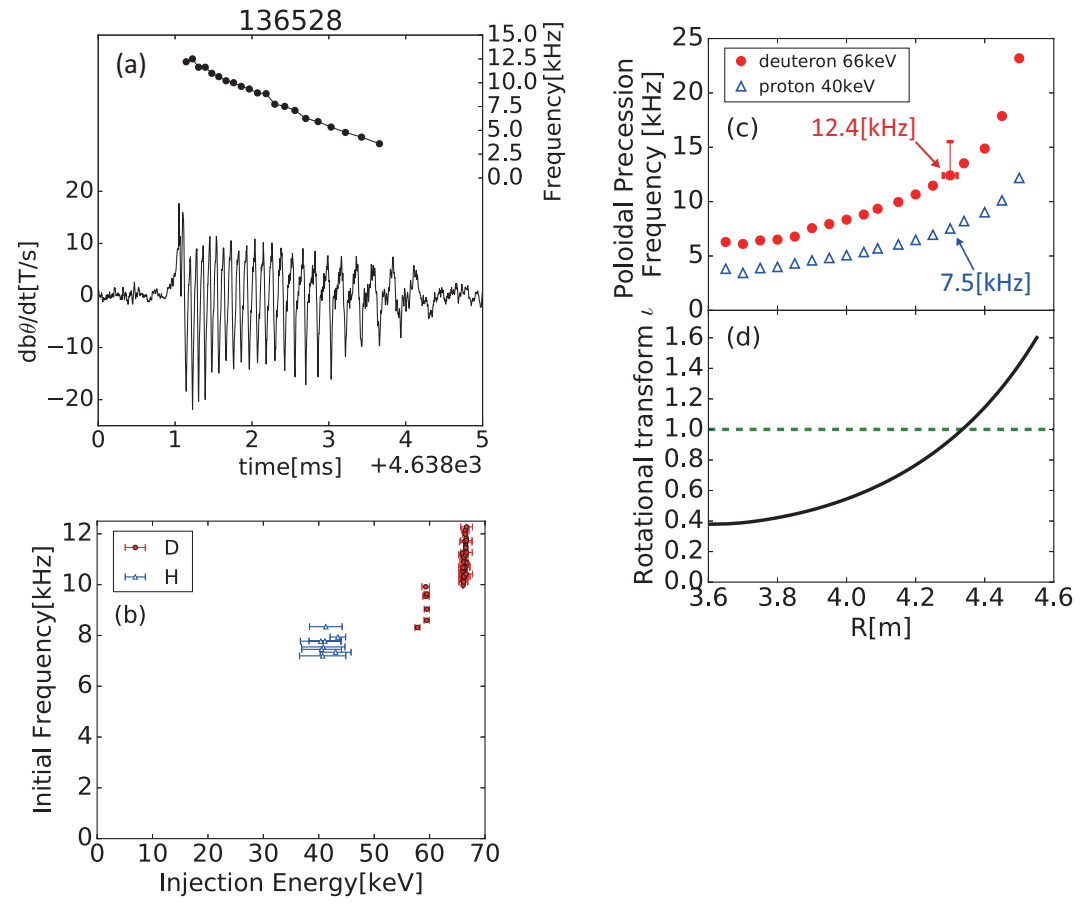

Figure 5. (a) Magnetic fluctuations induced by an EIC. The frequency chirping down is observed. (b) Observed initial frequency of magnetic fluctuations induced by EICs in different injection energies. (c) The poloidal precession frequencies estimated by calculating orbits of EPs regarding ions with $40 \mathrm{keV}$ and deuterons with $66 \mathrm{keV}$. (d) The $\iota$ profile of the vacuum configuration calculated by VMEC code [25]. 
Excitation of helically-trapped-energetic-ion driven resistive interchange modes with intense deuterium beam

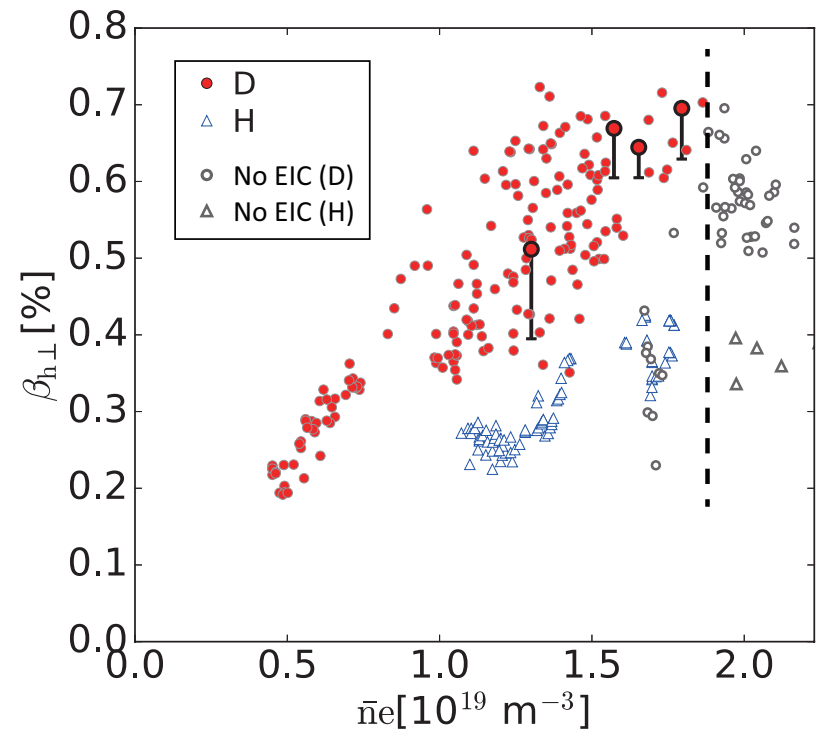

Figure 6. Relationship between $\beta_{\mathrm{h} \perp}$ and the line averaged electron density when EICs are excited with hydrogen (blue triangles) and deuterium (red dots) plasmas. The grey circles and grey triangles in the higher density region indicate data when EICs are not excited with deuterium and hydrogen PERP-NBIs, respectively. If $\bar{n}_{\mathrm{e}}>0.97 \times 10^{19} \mathrm{~m}^{-3}$, the deuterium injection powers are around $17 \mathrm{MW}$ without the case of $\beta_{\mathrm{h} \perp}<0.55 \%$ about $\bar{n}_{\mathrm{e}} \sim 1.7 \times 10^{19} \mathrm{~m}^{-3}$ and hydrogen injection powers are around $8 \mathrm{MW}$. If $\bar{n}_{\mathrm{e}}<0.97 \times 10^{19} \mathrm{~m}^{-3}$, the deuterium injection powers are around 8.5 MW. The length of vertical bars with some red dots indicate the decrease of $\beta_{\mathrm{h} \perp}$ assuming the experimentally obtained $T_{\mathrm{i}}$ and $T_{\mathrm{e}}$ profiles such as profiles in figure 3 . Note that contributions of TAN-NBIs to $\beta_{\mathrm{h} \perp}$ seems to be small compared to that of PERP-NBIs according to a previous simulation study [23]. 
Excitation of helically-trapped-energetic-ion driven resistive interchange modes with intense deuterium beam

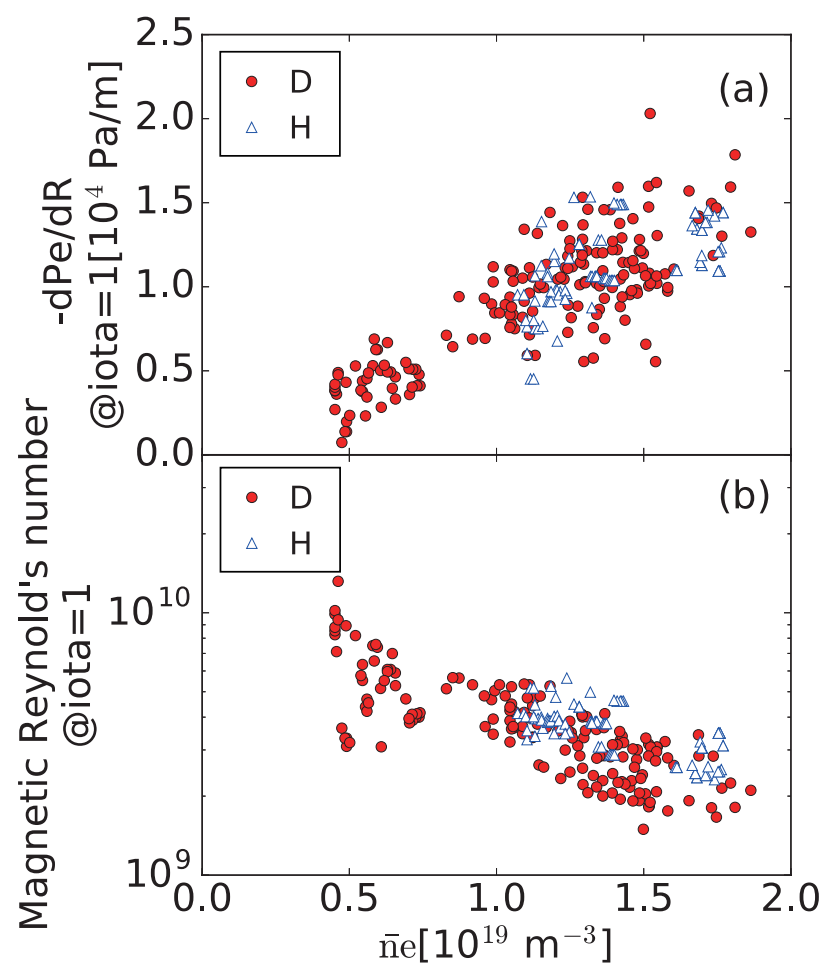

Figure 7. Comparison of (a) the electron pressure gradient and (b) the magnetic Reynold's number when EICs are excited with hydrogen (blue) and deuterium (red) plasmas.

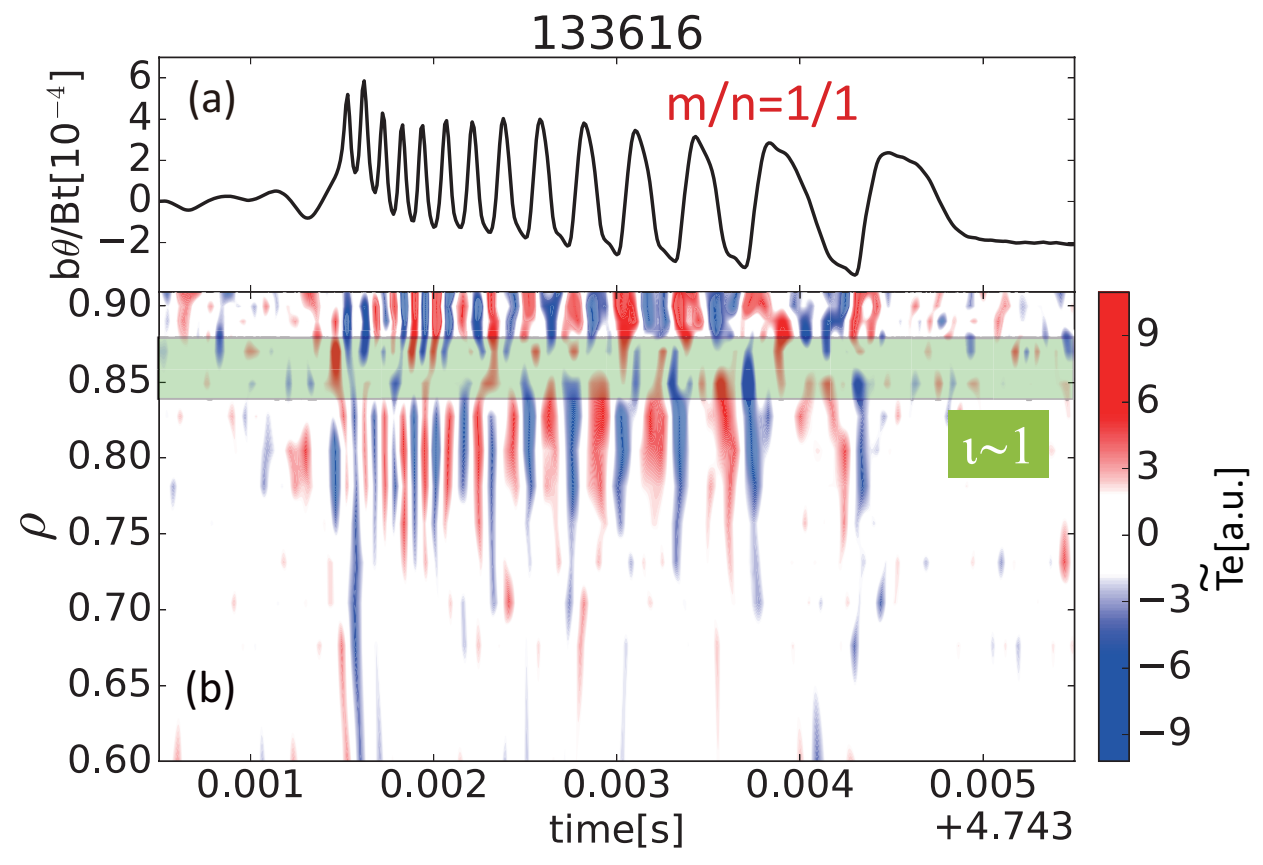

Figure 8. Time evolution of (a) magnetic fluctuations and (b) the profile of fluctuations of the electron temperature measured by ECE measurement [28]. $\rho$ is the normalized minor radius. 
Excitation of helically-trapped-energetic-ion driven resistive interchange modes with intense deuterium beam

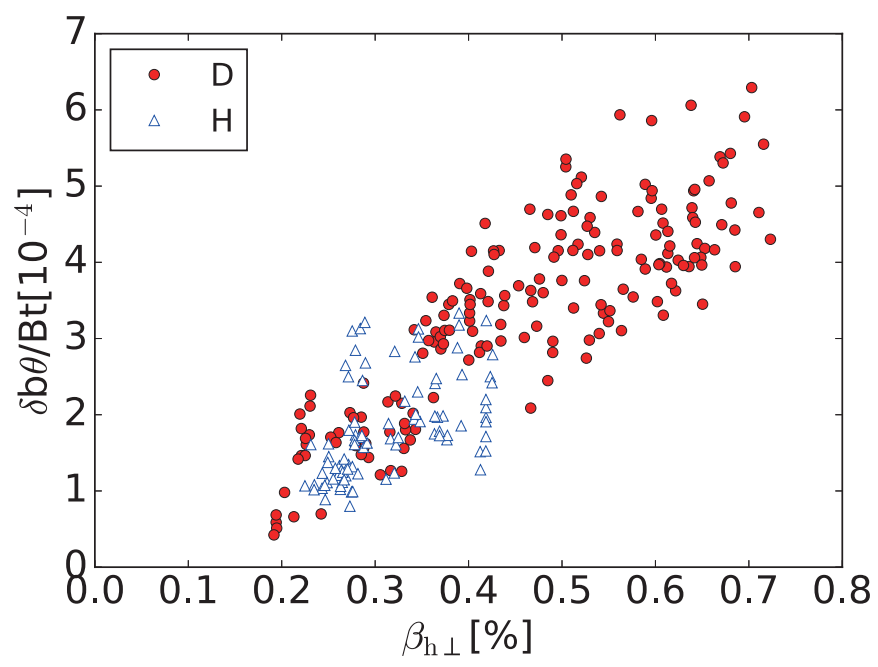

Figure 9. Relationship of $\beta_{\mathrm{h} \perp}$ and magnetic fluctuations when EICs are excited with hydrogen (blue) and deuterium (red) plasmas.

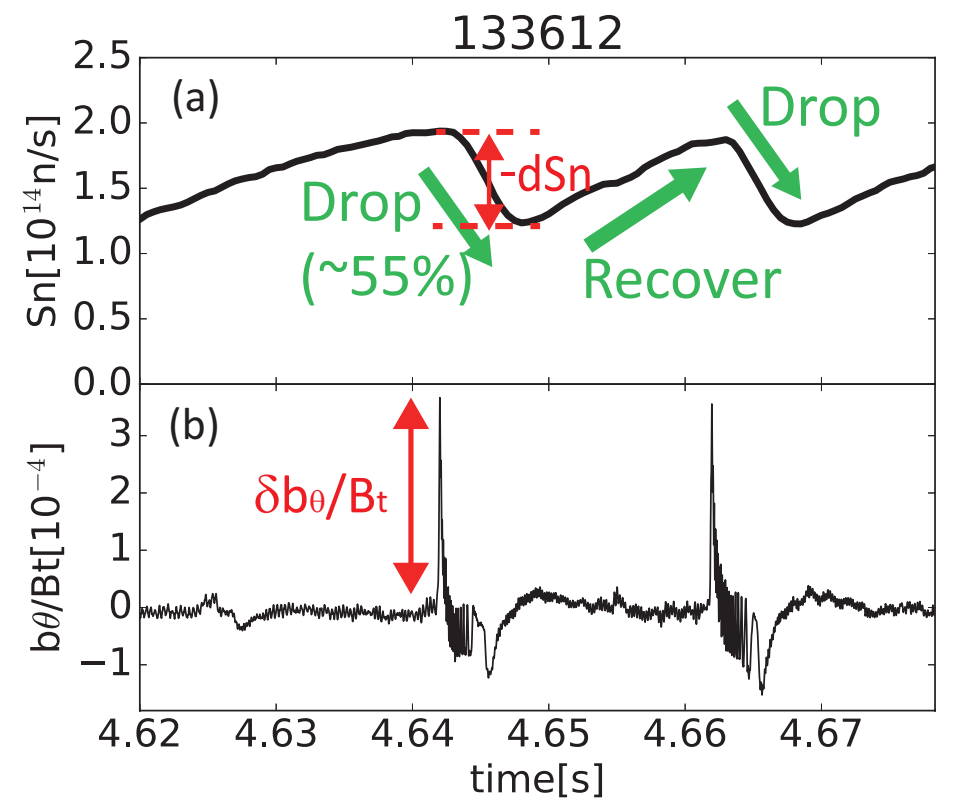

Figure 10. Time evolution of (a) the total neutron emission rate and (b) magnetic fluctuations. When EICs occur, the drop of the total neutron emission rate is observed. 
Excitation of helically-trapped-energetic-ion driven resistive interchange modes with intense deuterium beam

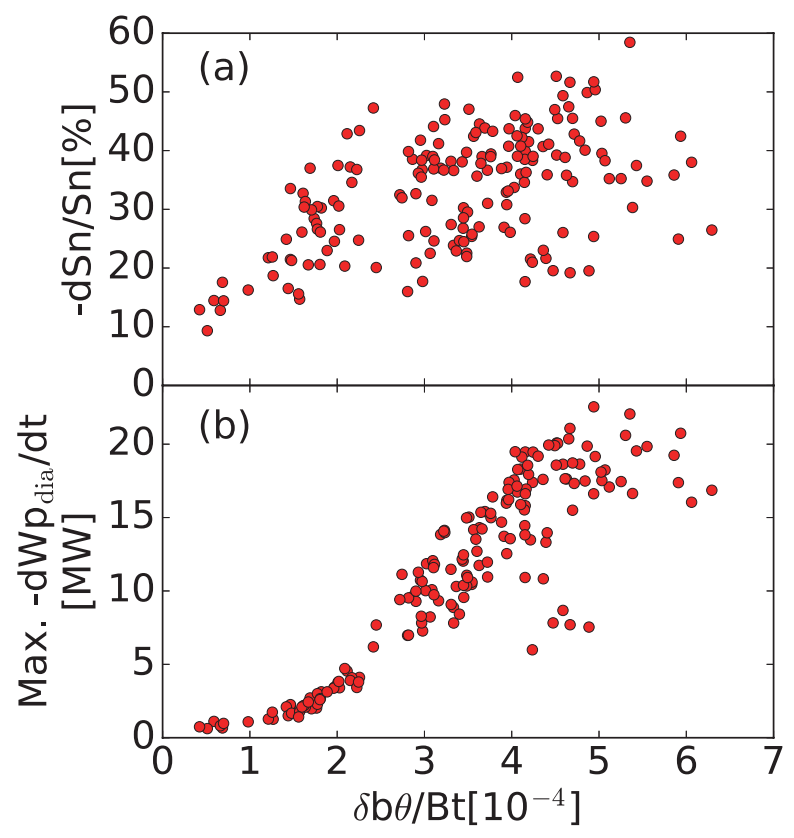

Figure 11. (a) Relationship of the drop rate of the total neutron emission rate with EICs and $\delta b_{\theta} / B_{\mathrm{t}}$. (b) Relationship of the maximum of $-d W p_{\text {dia. }} / d t$ just after EICs and $\delta b_{\theta} / B_{\mathrm{t}}$. Here, $d W p_{\text {dia. }} / d t$ is the time derivative of the plasma stored energy measured by the diamagnetic loop [19]. 
Excitation of helically-trapped-energetic-ion driven resistive interchange modes with intense deuterium beam
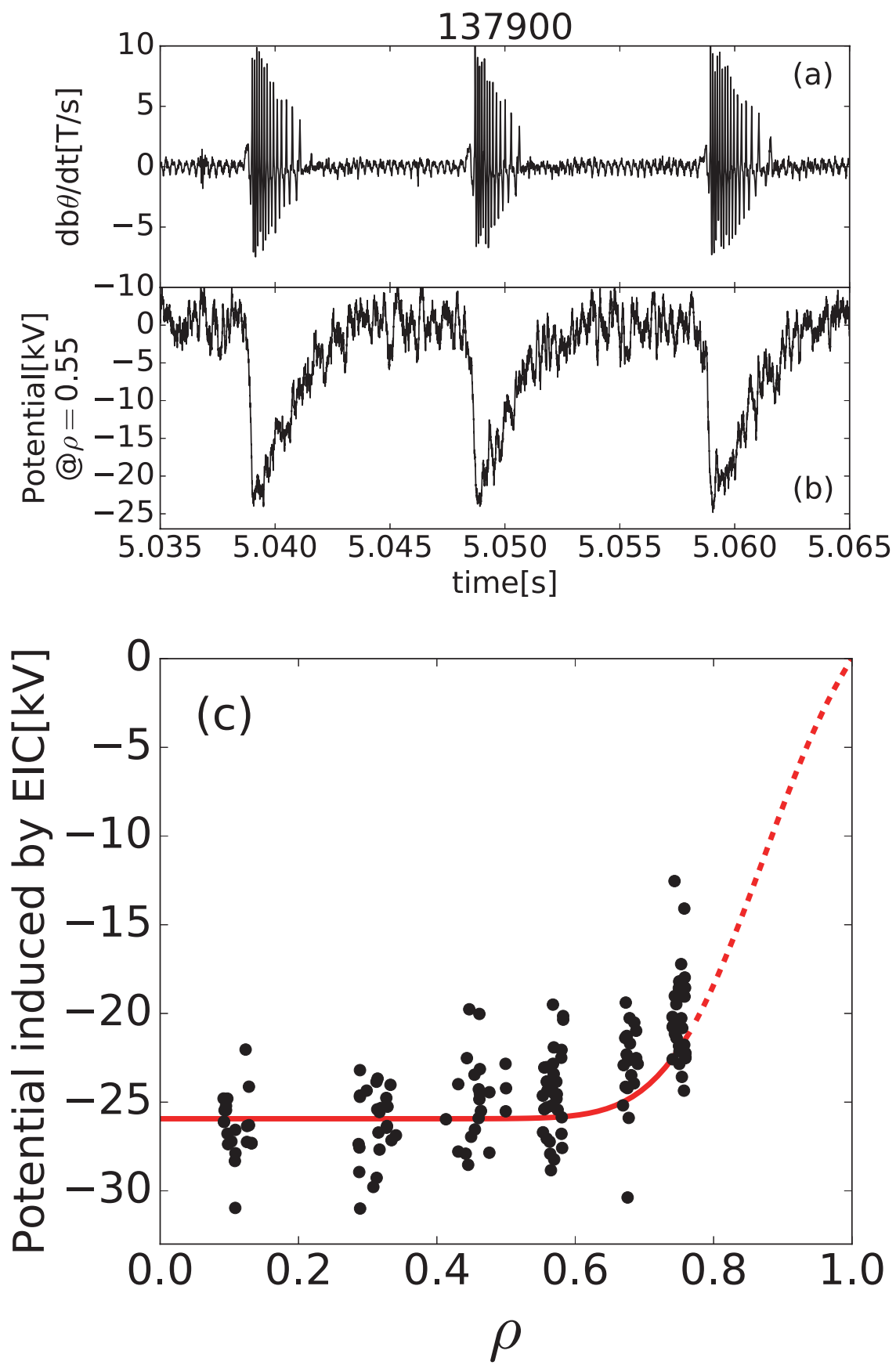

Figure 12. Figure (a) and (b) show time evolution of magnetic fluctuations and the electrostatic potential, respectively. Figure (c) shows the electrostatic potentials in different radial positions obtained with HIBP measurement [32]. $\rho$ is the normalized minor radius. The solid and dashed red lines are calculated by integrating $E_{r}=$ $-a \exp \left\{-\left[\frac{\rho-0.85}{0.15}\right]^{2}\right\} \mathrm{kV} \mathrm{m}^{-1}$ along the radius, which is as same as the function used in the reference [15]. $a$ is a factor which determines the depth of the potential. The dashed red line indicates the extrapolation assuming the potential increases monotonically. 
Excitation of helically-trapped-energetic-ion driven resistive interchange modes with intense deuterium beam

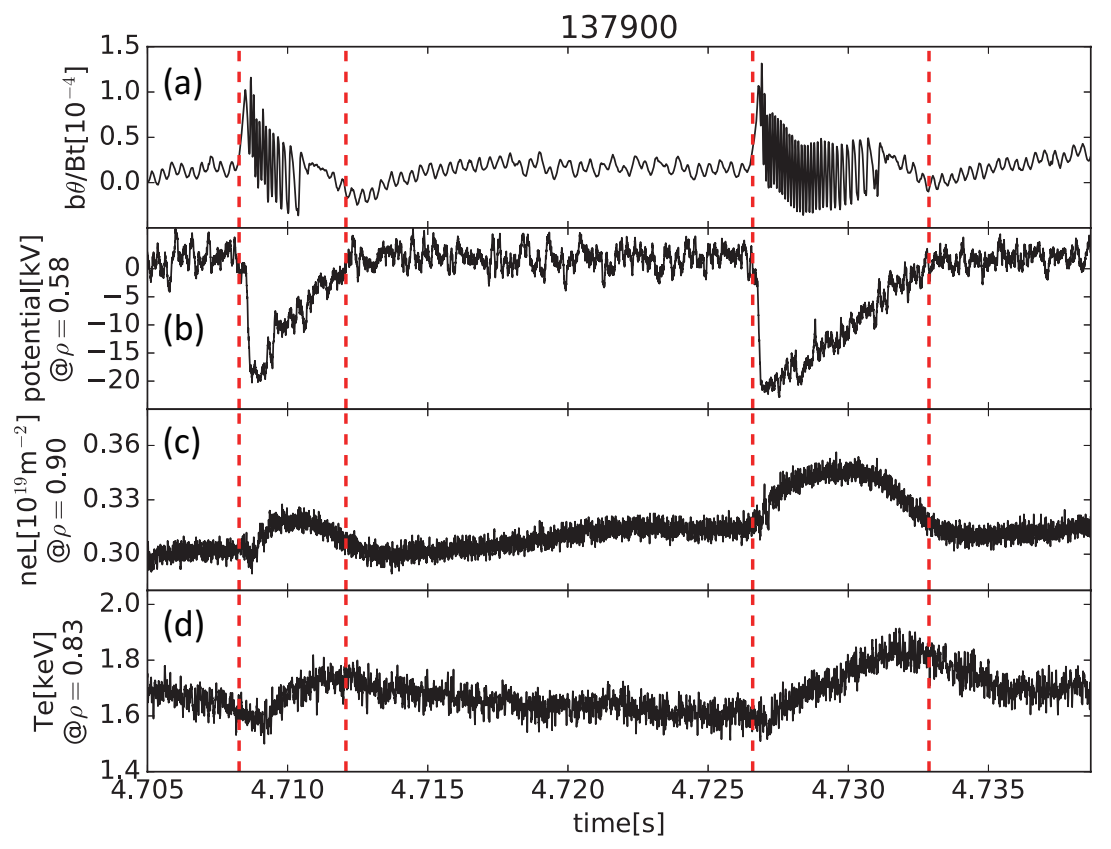

Figure 13. Time evolution of (a) magnetic fluctuations, (b) the electrostatic potential, (c) the line integrated electron density and (d) the electron temperature. The increase of the electron density and the electron temperature in the edge region are clearly observed when the electrostatic potential is formed. 